\title{
Missed nursing care, non-nursing tasks, staffing adequacy, and job satisfaction among nurses in a teaching hospital in Egypt
}

\author{
Marwa Hammad', Wafaa Guirguis ${ }^{2}$ and Rasha Mosallam² ${ }^{2^{*}}$
}

\begin{abstract}
Background: Missed nursing care (MNC) has been linked to patient harm in a growing body of literature. However, this issue is still not adequately investigated in developing countries. The aim of the study is to measure the extent of missed nursing care, to identify its types, and to determine factors contributing to missed nursing care.

Methods: A cross-sectional design was used. The study was conducted among 50 units at 1762-beds teaching Hospital in Alexandria that employs 1211 nurses in inpatient areas. A sample of 553 nurses were interviewed using the MISSCARE and the N4CAST survey. The MISSCARE survey measured the amount of missed nursing care (MNC) that was experienced on the last worked shift by each nurse. The N4CAST survey was used to collect data about level of non-nursing work carried out by nurses and the nurses' job satisfaction.

Results: The overall mean score for the missed nursing care was $2.26 \pm 0.96$ out of 5 , with highest mean score attributed to "Planning" and lowest mean score attributed to "Assessment and Vital Signs" (2.64 and 1.96, respectively). Missed nursing care was significantly associated with number of patients admitted and cared for in the last shift and perceived staffing adequacy. Almost all non-nursing care tasks and most of satisfaction elements showed negative weak correlation with overall missed nursing care.

Conclusion: Missed Nursing Care is common in study hospital which may endanger patient safety. MNC Missed Nursing Care is positively associated with nursing adequacy. There is no association between MNC and neither nurses' job satisfaction nor non-nursing tasks. Nursing leaders should monitor missed nursing care and the environmental and staffing conditions associated with it in order to design strategies to reduce such phenomena.
\end{abstract}

Keywords: Missed nursing care, Staffing adequacy, Non-nursing tasks, Egypt

\section{Introduction}

The term "missed nursing care" (MNC), introduced in 2006 [1], has been defined as "any aspect of required patient care that is omitted (either in part or in whole) or delayed" [2]. In a systematic review of 42 studies, four studies concluded that MNC is a

\footnotetext{
* Correspondence: rashaazm@yahoo.com

${ }^{2}$ High Institute of Public Health, Alexandria University, 165 El-Horeya Road, Al Ibrahimeyah Qebli WA Al Hadrah Bahri, Qism Bab Sharqi, Alexandria

Governorate, Egypt

Full list of author information is available at the end of the article
}

significant problem in hospitals; $55-98 \%$ of nurses reporting missing one or more items of required care during the time of assessment (frequently the last shift worked) [3]. The most frequently identified missed items were ambulating and turning patients, mouth care, feeding patients on time, comfort talk with patient and family, patient teaching, medication administration on time, and documentation [4-6].

Studies investigating reasons for MNC, as mentioned by nurses, concluded that inadequate labor resources was the most frequently cited reason, 
followed by material resources then communication. The most frequently reported items in labor resources were unexpected rise in patient volume and/ or acuity and inadequate number of staff, those in material resources were unavailability of medications when needed, and unavailability of supplies and equipment, while the most common items reported in communication were unbalanced patient assignments, and breakdowns in communication with medical staff $[4,6-8]$.

Studies examining the relationship between nurse staffing and MNC, used different staffing measures, and reported inconsistent findings. Using the objective measures of hours per patient day (HPPD), registered nurse hours per patient day, and skill mix, one study conducted in 10 hospitals in the USA identified HPPD as the only significant predictor of MNC [9], while another study in 11 hospitals in the USA reported that none of the measures was a significant predictor [5], and a third study in two hospitals in the USA reported that these measures were not predictors of patient-reported MNC [10]. Another study comparing MNC in high- vs. lowstaffing units in South Korea revealed that nurses working in high-staffing units had a significantly lower mean score of MNC than those in low-staffing units [11]. Nursing perceived level of adequate staffing was found to be a significant independent predictor in a study conducted in the USA, where those who perceived their staffing as adequate more often reported less MNC [4], while the same measure was not a significant predictor in another study conducted in eight hospitals in Iceland [12]. Four studies, using patient-to-nurse ratio as a measure of staffing, reported conflicting findings. Two studies, one conducted in 488 hospitals across 12 European countries and the other conducted in 10 acute care hospitals in the USA, concluded that higher patient-tonurse ratio was a significant predictor of $\mathrm{MNC}[4,13]$, while another study reported that a higher patient-tonurse ratio was a significant predictor of less $\mathrm{MNC}$ in an Italian medical care setting [6], and a fourth study conducted in Lebanon revealed that the patient-to-nurse ratio was not an independent predictor of MNC [14].

Several studies identified nurses spending their time performing non-nursing tasks (NNT) as an important reason for MNC. NNT include all tasks that are not related to direct patient care or tasks not requiring professional nursing skills [15-17]. In a study conducted in 55 private hospitals and seven public hospitals in South Africa, nursing tasks left undone were related to three non-nursing tasks, namely, "delivering and retrieving food trays," "routine phlebotomy/blood drawing for tests," and "cleaning patients' rooms and equipment" [17].

As regards the impact of MNC on nurses, a study conducted in 10 hospitals in the USA identified MNC as a significant predictor of intention to leave, but not of turnover [18]. Another study in the USA identified $\mathrm{MNC}$ as a significant predictor of job satisfaction; nurses who reported less MNC were more satisfied in their current position and occupation [19].

Literature search identified four Egyptian studies on MNC; all conducted in critical care units, and based on a small sample of nurses [20-23]. This research is a hospital-wide study to identify level and types of MNC and to determine factors contributing to missed nursing care, so that appropriate interventions can be developed to reduce care omissions and thus improve quality and safety of care.

\section{Methods}

\subsection{Study design and setting}

The study was conducted among 50 units at 1762 beds teaching hospital that employs 1211 nurses in inpatient areas. A cross-sectional design was used. The study population was nurses working in inpatient units who have been working in the hospital for a minimum of 3 months.

\subsection{Sample}

The following assumptions were used to calculate the required sample size of nurses to estimate the mean of MNC score: $95 \%$ confidence interval for mean \pm 0.1 and standard deviation of 1.2 [20]. The minimum required sample size is 553 nurses $\left(1.96^{*} 1.2 / 0.1\right)$. Units were randomly selected from all hospital inpatient units, and all nurses who fulfilled the inclusion criteria in each selected unit were invited to participate using convenient sampling, until the required sample size was reached. Inclusion criteria were nurses working in inpatient units of the study hospital who have been working in the hospital for a minimum of 3 months.

\subsection{Data collection}

A structured interview schedule was prepared to collect data from nurses about level of overall and specific elements of MNC, unit characteristics, nurse characteristics, nurse staffing measures, and nurse outcome measures.

The interview schedule consisted of two tools, the MISSCARE survey and the N4CAST survey. The MISS CARE survey [24] measures the amount of MNC that was experienced on the last worked shift by each respondent. It is composed of 24 items, belonging to four areas as follows: assessment and vital signs (8 items); interventions and individual needs (6 items); intervention/ basic needs ( 7 items); and planning ( 3 items). For each item, respondents are asked to identify the frequency of care being missed using a 5-point Likert scale from never missed to always missed. The mean of all 24 items 
is used as a total score for the scale, and the potential range of scores is 1 to 5 , with higher scores indicating more MNC. The questionnaire also collects data about work and demographic characteristics of nurses. The N4CAST survey [25] was used to collect data about level of NNT carried out by nurses. This section contains a list of nine tasks, and each nurse was required to indicate the frequency with which she/he performed each task during the most recent shift, on a 3-point scale of never, sometimes, and often (scored as 0,1 and 2, respectively). The items for NNT were the following: delivering food trays, performing non-nursing care, arranging discharge referrals and transportation, phlebotomy, transporting patients within the hospital, cleaning patients' rooms, filling-in for non-nursing services, obtaining supplies, and performing clerical duties. The N4CAST survey was also used to measure level of nurses' job satisfaction. Each nurse was required to indicate the level of satisfaction with the current job on a 4point scale of very dissatisfied, dissatisfied, satisfied, and very satisfied (scored as 1, 2, 3, and 4, respectively). The items for satisfaction were the following: work schedule flexibility, opportunities for advancement, independence at work, professional status, salary, educational opportunities, and annual sick and study leaves.

The study tools were translated to Arabic by the research team and then reviewed by a professional translator. The tool was assessed for face validity by a panel of experts. The questionnaire was piloted on 20 nurses to assure understandability and relevance of items. There were no changes in the tool and the 20 questionnaires were included in the sample.

\subsection{Statistical analysis}

Data was entered and analyzed using SPSS (Statistical Package for Social Sciences) version 21. Descriptive statistics (frequencies, percentages, and means) were performed. $T$ test was used for comparison of means between two groups and one-way ANOVA for comparison of means of more than two groups. The 5\% level was used as a cut-off point value for statistical significance. Spearman rank-order correlation was used to assess the correlation between $\mathrm{MNC}$ and non-nursing tasks and nurses' job satisfaction. Spearman rank-order correlation was selected as the data was not normally distributed.

\subsection{Ethical considerations}

Approvals of Ethics Committee of High Institute of Public Health and the Director of Central Administration of Alexandria Hospitals were obtained. Verbal consent was obtained from participants in the study, who were assured of confidentiality and anonymity of information and that it will be used for research purposes only. The researchers declare no conflict of interest.

\section{Results}

The majority of the study sample were females (94.4\%), in the age category $30<50$ years $(60.6 \%)$, working in surgical units (60.6\%), holding a nursing diploma degree (68.0\%), and working as a "Practical Nurse" (66.5\%).

As for workload of nurses at study hospital, the majority of nurses worked for more than $30 \mathrm{~h}$ per week $(89.0 \%)$ and cared for more than 10 patients per shift (43.4\%) with a mean of 11.94 patients. Highest percentage of the nurses had 0-5 patients admitted and discharged during the last shift $(84.6 \%$ and 89.5\%). As for perceived staff adequacy, around one-third of the sample perceived the staff to be adequate $50 \%$ of time and $16.1 \%$ perceived that the staff was never adequate.

The overall mean score for the MNC was 2.26 with highest mean score attributed to "Planning" and lowest mean score attributed to "Assessment and Vital Signs" (2.64 and 1.96, respectively). Item with the highest mean scores was attending "conferences" (2.84), whereas items with the least mean score was "IV site care," "Vital signs assessed as ordered(1.74 \pm 1.32$)$," and "Full documentation of all necessary data" ) $(1.76 \pm 1.36)$ (Table 1$)$.

MNC was not significantly associated with any of the work and demographic characteristics except the unit where the ICU was the unit with the lowest mean score as compared to the medical and surgical units (1.91 versus 2.48 and 2.30, respectively) (Table 2).

MNC was significantly associated with number of patients admitted and cared for in the last shift and perceived staffing adequacy. The higher the patients admitted and cared for the last shift, and the lower the perceived staff adequacy, the higher the overall MNC score (Table 3).

Unexpectedly, almost all non-nursing care tasks showed negative weak correlation with overall MNC. This means that the more the non-nursing tasks, the lower the overall MNC score (Table 4).

Most of satisfaction elements showed negative weak correlation with the overall MNC. This means that the higher the satisfaction, the lower the overall MNC score (Table 5).

\section{Discussion}

The overall MNC in the current study is much higher than a study comparing the MNC in the USA and Lebanon (2.26 for the current study versus 1.71 and 1.31 in the USA and Lebanon, respectively) [14]. Similarly, in another study conducted in a public 
Table 1 Mean scores of missed nursing care among inpatient nurses in a teaching hospital in Alexandria, Egypt

\begin{tabular}{|c|c|}
\hline Elements of MISSCARE survey & Total $(n=553)$ \\
\hline Assessment and vital signs (overall) & $1.96 \pm 1.07$ \\
\hline Full documentation of all necessary data & $1.76 \pm 1.36$ \\
\hline IV site care and assessment according to hospital policy & $1.70 \pm 1.31$ \\
\hline Monitoring intake/output & $1.90 \pm 1.30$ \\
\hline Vital signs assessed as ordered & $1.74 \pm 1.32$ \\
\hline Focused reassessment according to patient condition & $2.47 \pm 1.54$ \\
\hline Hand washing & $1.99 \pm 1.43$ \\
\hline Bedside glucose monitoring as ordered & $1.88 \pm 1.38$ \\
\hline Patient assessments performed each shift & $2.26 \pm 1.55$ \\
\hline Interventions and individual needs (overall) & $2.19 \pm 0.98$ \\
\hline Assessing effectiveness of medications & $2.30 \pm 1.44$ \\
\hline PRN medication requests acted up on within 15 min & $2.06 \pm 1.41$ \\
\hline Medications administered within 30 min before or after scheduled time & $1.96 \pm 1.28$ \\
\hline Assist with toileting needs within 5 min of request & $2.44 \pm 1.40$ \\
\hline Response to call is provided within 5 min & $2.0 \pm 1.34$ \\
\hline Emotional support to patient and/or family & $2.41 \pm 1.47$ \\
\hline Intervention /basic needs (overall) & $2.48 \pm 1.17$ \\
\hline Ambulation three times per day or as ordered & $2.72 \pm 1.48$ \\
\hline Turning patient every $2 \mathrm{~h}$ & $2.44 \pm 1.49$ \\
\hline Mouth care & $2.64 \pm 1.56$ \\
\hline Feeding patient when the food is still warm & $2.58 \pm 1.53$ \\
\hline Patient bathing & $2.65 \pm 1.62$ \\
\hline Setting up meals for patients who feed themselves & $2.29 \pm 1.58$ \\
\hline Skin/wound care & $2.01 \pm 1.37$ \\
\hline Planning (overall) & $2.64 \pm 1.08$ \\
\hline Patient teaching & $2.59 \pm 1.36$ \\
\hline Attending interdisciplinary care conferences whenever held & $2.84 \pm 1.42$ \\
\hline Ensuring discharge planning & $2.50 \pm 1.52$ \\
\hline Overall missed care & $2.26 \pm 0.96$ \\
\hline
\end{tabular}

Score (1-5) with higher score indicating higher MNC level

hospital in South Korea, the overall MNC was 1.39 for high staffing units (7 patients per RN) versus 1.51 for low staffing units (17 patients per RN) [6]. However, the results were comparable to the overall mean scores in four public hospitals in Australia where the mean scores ranged from 2.02 in ICU units to 2.40 in the medical units [26].

The overall dimensions "assessment and vital signs" and "interventions and individual needs" had less missed opportunities when compared to the overall dimensions "basic needs" and "planning." This result is consistent with the results of other studies $[4,7,27]$. Possible explanations for the MNC items is that those tasks that respondents perceived as important (e.g., vital sign and glucose monitoring) and which did not require teamwork were less frequently missed. On the other hand, care items, which may be perceived as less serious, although linked to patients' outcomes, for example, mouth care and those that often require teamwork (such as ambulation) were more likely to be missed [7]. Another possible explanation is that least frequently reported elements of MNC as vital signs, monitoring intake/output, and glucose monitoring are routinely recorded in nursing documentation and hence recognized by others if the medical file was audited. Conversely, ambulation of patients, patients' bathing, mouth care, and turning patients are nursing duties not routinely recorded in nursing documentation, and there is less opportunity for others to perceive 
Table 2 Association between overall missed nursing care and demographic and work characteristics

\begin{tabular}{|c|c|c|c|}
\hline Characteristic & $\begin{array}{l}\text { Overall MNC } \\
\text { Mean } \pm \text { SD }\end{array}$ & Test of Sig. & $\mathbf{p}$ \\
\hline Sex & & 1.15 & 0.24 \\
\hline Male & $2.45 \pm 0.84$ & & \\
\hline Female & $2.24 \pm 0.84$ & & \\
\hline Age group (years) & & 1.70 & 0.14 \\
\hline$<20$ & $2.30 \pm 0.88$ & & \\
\hline 20 to $<30$ & $2.25 \pm 0.88$ & & \\
\hline 30 to $<40$ & $2.12 \pm 0.86$ & & \\
\hline 40 to $<50$ & $2.27 \pm 0.99$ & & \\
\hline$\geq 50$ & $2.43 \pm 1.11$ & & \\
\hline Unit & & $14.31^{*}$ & 0.000 \\
\hline Medical unit & $2.48 \pm 0.77$ & & \\
\hline Surgical unit & $2.30 \pm 0.87$ & & \\
\hline ICU & $1.91 \pm 1.06$ & & \\
\hline Professional qualification (highest degree) & & 1.42 & 0.22 \\
\hline Nursing diploma (high school equivalent) & $2.29 \pm 0.94$ & & \\
\hline Nursing technician diploma & $2.29 \pm 1.19$ & & \\
\hline BSN (bachelor's degree) & $2.04 \pm 0.75$ & & \\
\hline Postgraduate diploma & $1.94 \pm 0.78$ & & \\
\hline Master's degree or more & 2.70 & & \\
\hline Job title & & 1.22 & 0.30 \\
\hline Head nurse (head of unit) & $1.98 \pm 0.90$ & & \\
\hline Nurse supervisor (BSN) & $2.14 \pm 0.78$ & & \\
\hline Nurse technician & $2.29 \pm 1.19$ & & \\
\hline Practical nurse (high school equivalent) & $2.28 \pm 0.93$ & & \\
\hline Shift worked & & 1.80 & 0.14 \\
\hline Morning & $2.32 \pm 1.05$ & & \\
\hline Evenings & $2.26 \pm 0.92$ & & \\
\hline Nights & $1.94 \pm 0.65$ & & \\
\hline Rotates & $2.20 \pm 0.87$ & & \\
\hline
\end{tabular}

${ }^{*} P$ value $<0.05$

( $T$ test was used for comparison of means between two groups and one-way ANOVA for comparison of means of more than two groups)

this care as missed. Increased attention to these elements, including a refocus of existing documentation systems, is a necessity [27].

Hospital unit was significantly associated with the overall MNC score where the ICU had a lower overall MNC score when compared to the medical and surgical units (1.91 versus 2.48 and 2.30 , respectively) $(P<0.000)$. This result is consistent with the results of a study conducted in eight hospitals in Iceland and another study conducted in four hospitals in Australia and among ICU units in two teaching hospitals in Egypt [7, 12, 20]. The better scores in the ICU can be explained by the fact that ICU has skilled nursing staff who hold at least bachelor degree in nursing. In addition, the nurse to patient ratio is usually $1: 1$ or $1: 2$ in ICU which reduce the workload on nurses and hence reduce the missed care.

Higher mean scores of MNC were significantly associated with increasing number of patients cared for and admitted in the last shift and nurses' perceived inadequacy of staffing. Several studies conducted in the USA and South Korea, demonstrated the association between nurses' workload and staffing adequacy and MNC [11, 12, 26, 28]. In other studies, staffing adequacy had a positive impact on reducing instances of failure to rescue, inpatient mortality, postoperative mortality, and length of 
Table 3 Association between overall missed nursing care and staffing adequacy, workload, and intention to leave

\begin{tabular}{|c|c|c|c|c|c|}
\hline \multirow[t]{2}{*}{ Characteristic } & \multicolumn{2}{|c|}{ Total $(n=553)$} & \multirow{2}{*}{$\begin{array}{l}\text { Overall } \\
\text { MNC } \\
\text { Mean } \pm \\
\text { SD }\end{array}$} & \multirow{2}{*}{$\begin{array}{l}\text { Test } \\
\text { of } \\
\text { Sig. }\end{array}$} & \multirow[t]{2}{*}{$\mathbf{p}$} \\
\hline & No. & $\%$ & & & \\
\hline No. of working hours/week & & & & 0.69 & 0.48 \\
\hline$\geq 30 \mathrm{~h}$ & 492 & 89.0 & $2.17 \pm 0.99$ & & \\
\hline$<30 \mathrm{~h}$ & 61 & 11.0 & $2.26 \pm 0.96$ & & \\
\hline No. of patients cared for/last shift & & & & $17.49^{*}$ & 0.00 \\
\hline $0-5$ & 213 & 38.5 & 1.97 & & \\
\hline $6-10$ & 100 & 18.1 & 2.26 & & \\
\hline$>10$ & 240 & 43.4 & 2.49 & & \\
\hline Mean \pm SD & 11.94 & & & & \\
\hline No. of patient admissions/last shift & & & & $4.32^{*}$ & 0.01 \\
\hline $0-5$ & 468 & 84.6 & 2.20 & & \\
\hline $6-10$ & 78 & 14.1 & 2.54 & & \\
\hline$>10$ & 7 & 1.3 & 2.45 & & \\
\hline Mean \pm SD & 3.17 & & & & \\
\hline No. of patient discharges/last shift & & & & 2.27 & 0.10 \\
\hline $0-5$ & 495 & 89.5 & 2.27 & & \\
\hline $6-10$ & 49 & 8.9 & 2.23 & & \\
\hline$>10$ & 9 & 1.6 & 1.58 & & \\
\hline Mean \pm SD & 2.50 & & & & \\
\hline Perceived staffing adequacy & & & & $7.88^{*}$ & 0.00 \\
\hline $100 \%$ of the time & 47 & 8.5 & $2.27 \pm 1.00$ & & \\
\hline $75 \%$ of the time & 130 & 23.5 & $2.04 \pm 0.81$ & & \\
\hline $50 \%$ of the time & 158 & 28.6 & $2.07 \pm 0.80$ & & \\
\hline $25 \%$ of the time & 129 & 23.3 & $2.41 \pm 1.10$ & & \\
\hline $0 \%$ of the time & 89 & 16.1 & $2.64 \pm 1.03$ & & \\
\hline Work retention plan & & & & 1.65 & 0.09 \\
\hline Plan to leave work within 6 months & 137 & 24.8 & $2.38 \pm 1.15$ & & \\
\hline No plan to leave work within 6 months & 416 & 75.2 & $2.21 \pm 0.88$ & & \\
\hline
\end{tabular}

${ }^{*} P$ value $<0.05$

( $T$ test was used for comparison of means between two groups and one-way ANOVA for comparison of means of more than two groups)

Table 4 Spearman rank-order correlations between overall missed nursing care and non-nursing tasks

\begin{tabular}{|c|c|c|}
\hline Non-nursing task & $\begin{array}{l}\text { Total }(n=553) \\
\text { Mean }(S D)^{\mathrm{a}}\end{array}$ & Overall MNC \\
\hline Delivering and retrieving food trays & $0.79(0.81)$ & $-0.133^{*}$ \\
\hline Performing non-nursing care & $1.14(0.70)$ & -0.017 \\
\hline Arranging discharge referrals and transportation (including long-term care) & $1.43(0.68)$ & -0.077 \\
\hline Routine phlebotomy/blood draw for tests & $1.72(0.53)$ & -0.041 \\
\hline Transporting patients within hospital & $1.36(0.65)$ & $-0.085^{*}$ \\
\hline Cleaning patient rooms and equipment & $1.45(0.68)$ & $-0.259^{* *}$ \\
\hline Filling in for non-nursing services and available on off hours & $0.96(0.78)$ & 0.027 \\
\hline Obtaining supplies or equipment & $1.53(0.63)$ & $-0.085^{*}$ \\
\hline Answering phones, clerical duties & $0.79(0.81)$ & 0.056 \\
\hline
\end{tabular}

"Spearman correlation coefficient $P$ value $<0.05$

** Spearman Correlation Coefficient $P$ value $<0.01$

${ }^{\text {aScores }(0-2)}$ 
Table 5 Spearman rank-order correlations between missed nursing care and satisfaction

\begin{tabular}{lll}
\hline Satisfaction dimension & $\begin{array}{l}\text { Total }(\boldsymbol{n}=\mathbf{5 5 3}) \\
\text { Mean (SD) }\end{array}$ & Overall MNC \\
\hline Work schedule flexibility & $2.49 \pm 0.90$ & $-0.136^{* *}$ \\
Opportunity for advancement & $2.47 \pm 0.92$ & $-0.110^{* *}$ \\
Professional status & $2.49 \pm 0.96$ & $-0.186^{* *}$ \\
Salary & $1.82 \pm 0.88$ & $-0.103^{*}$ \\
Educational opportunities & $2.25 \pm 0.91$ & 0.015 \\
Annual leave & $2.26 \pm 0.90$ & 0.054 \\
Sick leave & $1.99 \pm 0.92$ & $-0.119^{* *}$ \\
Study leave & $2.05 \pm 0.91$ & -0.001 \\
Current job & $2.87 \pm 0.85$ & -0.066 \\
Independence at work, be a nurse or technician & $2.83 \pm 0.88$ & -0.095 \\
Team work and co-operation within department/unit & $2.89 \pm 0.88$ & -0.079 \\
Overall & $2.40 \pm 0.56$ & $-0.170^{* *}$ \\
\hline
\end{tabular}

"Spearman correlation coefficient $P$ value $<0.05$

** Spearman Correlation Coefficient $P$ value $<0.01$

${ }^{\mathrm{a} S}$ Scores (1-4)

hospital stay [29-31]. Thus, adequacy of staffing is of paramount importance in ensuring patient safety and reducing missed nursing care. Thus, it is recommended to use a staffing approach which accounts for individual patient variation in need as alternatives to, or in conjunction with, minimum staffing levels based entirely on patient volumes which is used in public hospitals in Egypt [32].

In the current study, only two non-nursing tasks correlated positively with missed nursing care: cleaning patients' rooms and filling in for non-nursing services and available on off hours. Both correlations were weak and non-significant. This unexpected finding could be explained by the fact that nurses may consider all the other non-nursing tasks to be part of their workload. Studies pointed out that nurses accept performing non-nursing duties, because these roles are founded on organizational as well as individual patient relationships [33].

The current study showed transporting patients in hospital correlated negatively and significantly with certain missed nursing care elements. The rationale for this might be that when patients are transferred outside of the unit, it is crucial that all procedures are done before leaving the unit [17].

Overall satisfaction showed a negative weak significant correlation with overall MNC. This is consistent with the results of two studies conducted in the USA where nurses who perceived less MNC on the patient care unit where they work are more satisfied in their current position and occupation [19, 34]. This can have two possible explanations in the present study. First, is that when nurses see that the elements of nursing care are missed, they become more dissatisfied. This can be explained by the fact that people respond positively and with more motivation to do a good job when they witness their constituents benefiting from their efforts. In the case of nursing, unlike some other occupations, the providers have direct and many times immediate knowledge about the effect of the quality of their work on their patients. Thus, nurses are fully cognizant of the impact of missing care for their patients, and when the effect is negative $[19,35]$. This was supported by a study conducted in the USA where each one unit increase in missed nursing care was associated with a 0.26 decrease in job enjoyment [36]. The second possible explanation is that the dissatisfied nurse is more prone to miss elements of nursing care. Further research should explore the causative relation between MNC and nurses' satisfaction.

\subsection{Limitations}

The current study addresses the concept of MNC in one of the largest teaching hospitals in Egypt. It sets a basis for further assessments and suggestions for improvement. Further research studying the link between MNC and adverse events is recommended.

The current study used subjective measures of missed care, with most relying on retrospective reports by nurses. Further studies using objective measures as direct observation of nursing care are recommended to evaluate the current study findings. The study was conducted in a teaching hospital which limits the generalization of the results to other settings as private, Ministry of Health and Insurance settings.

\section{Conclusions}

The missed nursing care is relatively higher when compared to other settings. The dimensions which are 
frequently missed are "basic needs" and "planning." The mean missed care was lower in the ICU as compared to the medical and surgical units. Nursing staffing adequacy and number of patients cared by nurse were factors associated significantly with higher score of missed nursing care. Non-nursing tasks and nurses' job satisfaction showed negative weak correlation with the overall MNC.

\section{Abbreviation \\ MNC: Missed nursing care}

\section{Acknowledgements}

The authors would like to express their gratitude for the nurses who agreed to conduct the interview and the hospital administration who facilitated the conduction of the research.

\section{Authors' contributions}

$\mathrm{MH}$ has made substantial contributions to or acquisition and interpretation of data. WG has made substantial contributions to conception and design, analysis, and interpretation of data. RM has contributed to analysis and interpretation of data and drafted the manuscript. The authors read and approved the final manuscript.

\section{Funding}

There was not any funding agency for this research.

\section{Availability of data and materials}

The data are available from the corresponding author on reasonable request.

\section{Declarations}

\section{Ethics approval and consent to participate}

The manuscript was approved by the ethics committee of the High Institute of Public Health, Alexandria University and the Director of Central Administration of Alexandria Hospitals (The Ethics Committee does not provide a reference number). Verbal consent was obtained from nurses. The ethics committee approved this type of consent. The purpose of the study was explained to the nurses and they were assured of confidentiality and anonymity of information and that it will be used for research purposes only. Verbal consent was approved by the ethics committee. Following this verbal explanation, a study information sheet was given to the nurses and they were allowed enough time to consider whether or not to participate in the study.

Procedures for the ensuring nurses confidentiality were taken. Identification code numbers were used on the questionnaires instead of personal identifiers. Access to research data was limited to the research team.

\section{Consent for publication}

Not applicable.

\section{Competing interests}

Dr. Rasha Mosallam is a co-author of this study and editorial board member of the journal. She declares a competing interest for this submission. She has not handled this manuscript. The rest of the authors have no conflict of interest to declare.

\section{Author details}

'Ministry of Health, 97 El-Horeya Road, Qism Bab Sharqi, Wabour Al Meyah, Alexandria Governorate, Egypt. ${ }^{2}$ High Institute of Public Health, Alexandria University, 165 El-Horeya Road, Al Ibrahimeyah Qebli WA Al Hadrah Bahri, Qism Bab Sharqi, Alexandria Governorate, Egypt.

Received: 22 July 2020 Accepted: 22 June 2021

Published online: 20 July 2021

\section{References}

1. Kalisch BJ. Missed nursing care: a qualitative study. J Nurs Care Qual. 2006; 21(4):306-13.
2. Kalisch BJ, Landstrom GL, Hinshaw AS. Missed nursing care: a concept analysis. J Adv Nurs. 2009;65(7):1509-17.

3. Jones TL, Hamilton P, Murry N. Unfinished nursing care, missed care, and implicitly rationed care: state of the science review. Int J Nurs Stud. 2015; 52(6):1121-37.

4. Kalisch BJ, Tschannen D, Lee H, Friese CR. Hospital variation in missed nursing care. Am J Med Qual. 2011;26(4):291-9.

5. Kalisch BJ, Lee KH. Missed nursing care: magnet versus non-magnet hospitals. Nurs Outlook. 2012;60:e32-9.

6. Palese A, Ambrosi E, Prosperi L, Guarnier A, Barelli P, Zambiasi P, et al. Missed nursing care and predicting factors in the Italian medical care setting. Intern Emerg Med. 2015;10(6):693-702.

7. Chapman R, Rahman A, Courtney M, Chalmers C. Impact of teamwork on missed care in four Australian hospitals. J Clin Nurs. 2017;26(1-2):170-81.

8. Moreno-Monsiváis MG, Moreno-Rodríguez C, Interial-Guzmán MG. Missed nursing care in hospitalized patients. Aquichan. 2015;15(3):318-28.

9. Kalisch BJ, Tschannen D, Lee KH. Do staffing levels predict missed nursing care? Int J Qual Health Care. 2011;23(3):302-8.

10. Dabney BW, Kalisch BJ. Nurse staffing levels and patient-reported missed nursing care. J Nurs Care Qual. 2015;30(4):306-12.

11. Kim YS, Yeon KN, You SJ, Lee ID. Effects of increasing nurse staffing on missed nursing care. Int Nurs Rev. 2015;62:267-74.

12. Bragadóttir H, Kalisch BJ, Tryggvadóttir GB. Correlates and predictors of missed nursing care in hospitals. J Clin Nurs. 2017;26(11-12):1524-34.

13. Ausserhofer D, Zander B, Busse R, Schubert M, De Geest S, Rafferty AM, et al. Prevalence, patterns and predictors of nursing care left undone in European hospitals: results from the multicountry cross-sectional RN4CAST study. BMJ Qual Saf. 2014;23(2):126-35.

14. Kalisch BJ, Doumit M, Lee KH, Zein JE. Missed nursing care, level of staffing, and job satisfaction: Lebanon versus the United States. J Nurs Adm. 2013; 43(5):274-9.

15. Al-Kandari F, Thomas D. Perceived adverse patient outcomes correlated to nurses' workload in medical and surgical wards of selected hospitals in Kuwait. J Clin Nurs. 2008:18:581-90.

16. Aiken LH, Clarke SP, Sloane DM, Sochalski JA, Busse R, Clarke H, et al. Nurses' reports on hospital care in five countries. Health Aff (Millwood). 2001;20(3): 43-53.

17. Bekker M, Coetzee SK, Klopper HC, Ellis SM. Non-nursing tasks, nursing tasks left undone and job satisfaction among professional nurses in South African hospitals. J Nurs Manag. 2015;23(8):1115-25.

18. Tschannen D, Kalisch BJ, Lee KH. Missed nursing care: the impact on intention to leave and turnover. Can J Nurs Res. 2010:42(4):22-39.

19. Kalisch B, Tschannen D, Lee H. Does missed nursing care predict job satisfaction? J Healthc Manag. 2011:56:117-31.

20. Abd Elrehem MAE, Abd El Fatah MA, Seloma YA. Correlates of missed nursing care in selected medical intensive care units, Egypt. J Biol Agric Healthc. 2014;4:88-105.

21. Attia NM, Abdeen MA, El Sayed SH. Impact of nursing teamwork on missed nursing care in intensive care units at Zagazig University Hospitals. Zagazig Nurs J. 2014;10(2):201-17.

22. Mohamed SA, Abed F. Perception of teamwork and missed nursing care among nurses in intensive care units at South Valley University Hospitals. IOSR J Nurs Health Sci. 2016:5:89-97.

23. El Rehem MAE, Elhamid MA, Elsalam YA. Missed nursing care: observation versus perception in selected medical intensive care units, Egypt. Int J Health Wellness Soc. 2017;7(1):31-47.

24. Kalisch BJ, Williams RA. Development and psychometric testing of a tool to measure missed nursing care. J Nurs Adm. 2009;39(5):211-9.

25. Report of the Irish RN4CAST study 2009-2011. [Cited 2017 Nov 8]. Available from: http://doras.dcu.ie/19344/1/RN4CAST_FINAL_report_18_April_2013_ DORAS.pdf

26. Orique SB, Patty CM, Woods E. MNC and unit-level nurse workload in the acute and post-acute settings. J Nurs Care Qual. 2016:31(1):84-9.

27. Kalisch BJ, Xie B. Errors of omission: missed nursing care. West J Nurs Res. 2014:36(7):875-90.

28. Tubbs-Cooley HL, Mara CA, Carle AC, Mark BA, Pickler RH. Association of nurse workload with missed nursing care in the neonatal intensive care unit. JAMA Pediatr. 2019;173(1):44-51.

29. Ball JE, Bruyneel L, Aiken LH, Sermeus W, Sloane DM, Rafferty AM, et al. RN4Cast Consortium. Post-operative mortality, missed care and nurse 
staffing in nine countries: a cross-sectional study. Int J Nurs Stud. 2018;78: 10-5.

30. Clarke SP, Aiken LH. Failure to rescue: needless deaths are prime examples of the need for more nurses at the bedside. Am J Nurs. 2003:103(1):42-7.

31. Kane RL, Shamliyan TA, Mueller C, Duval S, Wilt TJ. The association of registered nurse staffing levels and patient outcomes: systematic review and meta-analysis. Med Care. 2007:45(12):1195-204.

32. Griffiths P, Saville C, Ball J, Jones J, Pattison N, MonksT, et al. Safer Nursing Care Study Group. Nursing workload, nurse staffing methodologies and tools: a systematic scoping review and discussion. Int J Nurs Stud. 2019;103: 103487.

33. Allen D. Re-conceptualising holism in the contemporary nursing mandate: from individual to organisational relationships. Soc Sci Med. 2014;119:131-8.

34. Duffy JR, Culp S, Padrutt T. Description and factors associated with missed nursing care in an acute care community hospital. J Nurs Adm. 2018;48(7-8): $361-7$.

35. Grant AM. Relational job design and the motivation to make a prosocial difference. Acad Manag Rev. 2007;32(1):393-417.

36. Smith JG, Rogowski JA, Lake ET. Missed care relates to nurse job enjoyment and intention to leave in neonatal intensive care. J Nurs Manag. 2020;00:1-8.

\section{Publisher's Note}

Springer Nature remains neutral with regard to jurisdictional claims in published maps and institutional affiliations.

\section{Submit your manuscript to a SpringerOpen ${ }^{\odot}$ journal and benefit from:}

- Convenient online submission

- Rigorous peer review

- Open access: articles freely available online

High visibility within the field

- Retaining the copyright to your article

Submit your next manuscript at $\boldsymbol{\nabla}$ springeropen.com 